\title{
Facharztprüfung - was gilt? (Stand 2005)
}

\author{
C. Hänggeli, Geschäftsleiter Sekretariat Aus-, Weiter- und Fortbildung (AWF)
}

Der Zentralvorstand setzt per 1. Januar 2005 auch die Facharztprüfungen in Angiologie und pädiatrischer Endokrinologie-Diabetologie mit eliminatorischer Wirkung in Kraft.

\begin{tabular}{|c|c|c|c|c|c|}
\hline \multicolumn{2}{|c|}{$\begin{array}{l}\text { Tabelle } \\
\text { Muss ich die Facharztprüfung bestehen? } \\
\text { (Fortsetzung auf der folgenden Seite) } \\
\text { Facharzttitel Inkraftsetzungstermin }\end{array}$} & \multirow[t]{2}{*}{ 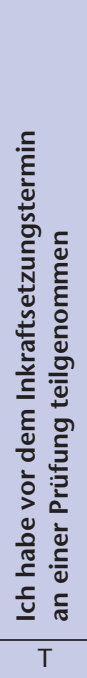 } & 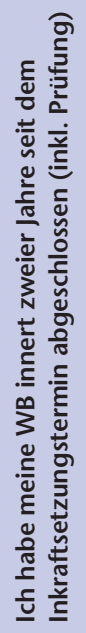 & 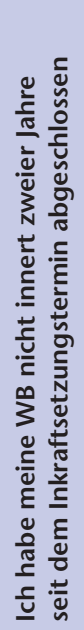 & 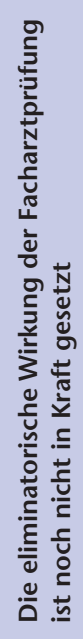 \\
\hline Allergologie und klinische Immunologie & 1.1 .2003 & & $\mathrm{~T}$ & $\mathrm{~B}$ & - \\
\hline Allgemeinmedizin & 1.1 .2000 & $T$ & $T$ & B & - \\
\hline Anästhesiologie & 2.4 .1986 & - & - & B & - \\
\hline Angiologie & 1.1 .2005 & $T$ & T & B & - \\
\hline Arbeitsmedizin & 1.1 .2001 & $T$ & $T$ & B & - \\
\hline Chirurgie (inkl. Basisexamen) & 1.1.1999 & $\mathrm{T}$ & $\mathrm{T}$ & B & - \\
\hline Dermatologie und Venerologie & 1.1 .2003 & T & T & B & - \\
\hline Endokrinologie/Diabetologie & 1.1 .2001 & $T$ & $\mathrm{~T}$ & B & - \\
\hline Gastroenterologie & 1.1 .2000 & $T$ & $T$ & B & - \\
\hline Gynäkologie und Geburtshilfe & 1.1 .1999 & $T$ & $T$ & B & - \\
\hline Hämatologie & 1.1 .2001 & $T$ & $T$ & B & - \\
\hline Herz- und thorakale Gefässchirurgie & $1.1 .2006 ?$ & - & - & - & $T$ \\
\hline Infektiologie & 1.1 .2003 & $T$ & $\mathrm{~T}$ & B & - \\
\hline Innere Medizin & 1.1 .1999 & $T$ & $T$ & B & - \\
\hline Intensivmedizin & 1.1 .2001 & $T$ & $T$ & B & - \\
\hline Kardiologie & 1.1.1999 & $T$ & $T$ & B & - \\
\hline Kiefer- und Gesichtschirurgie & $1.1 .2006 ?$ & - & - & - & $\mathrm{T}$ \\
\hline Kinderchirurgie & 2.4 .1986 & - & - & B & - \\
\hline Kinder- und Jugendmedizin & 1.1 .2004 & $T$ & $\mathrm{~T}$ & B & - \\
\hline $\begin{array}{l}\text { Kinder- und Jugendpsychiatrie } \\
\text { und -psychotherapie }\end{array}$ & 1.1 .2002 & $T$ & $T$ & B & - \\
\hline Klinische Pharmakologie und Toxikologie & $1.1 .2006 ?$ & - & - & - & $T$ \\
\hline Medizinische Genetik & $1.1 .2006 ?$ & - & - & - & $\mathrm{T}$ \\
\hline Medizinische Onkologie & 1.1 .2001 & $\mathrm{~T}$ & $\mathrm{~T}$ & B & - \\
\hline Nephrologie & $1.1 .2006 ?$ & - & - & - & $\mathrm{T}$ \\
\hline Neurochirurgie & 2.4 .1986 & - & - & B & - \\
\hline Neurologie & 1.1 .2003 & $\mathrm{~T}$ & $\mathrm{~T}$ & B & - \\
\hline Nuklearmedizin & 2.4 .1986 & - & - & B & - \\
\hline Ophthalmologie & 1.1 .2002 & $T$ & $T$ & B & - \\
\hline
\end{tabular}

Per 1. Januar 2005 hat der Zentralvorstand in zwei weiteren Fachgebieten die eliminatorische Wirkung der Facharztprüfung in Kraft gesetzt. Damit wird jetzt für praktisch alle Facharzttitel das Bestehen der Facharztprüfung gefordert, selbstverständlich unter Vorbehalt der anwendbaren Übergangsbestimmungen.

\section{Übergangsbestimmungen}

Ausnahmen gelten für Kandidatinnen und Kandidaten, welche eine der beiden folgenden Bedingungen erfüllen:

- Wer vor dem Inkraftsetzungstermin bereits an einer Facharztprüfung (ganz oder teilweise) teilgenommen hat, muss keine weitere Prüfung absolvieren.

- Wer die Weiterbildung innert zweier Jahre nach dem Inkraftsetzungstermin abgeschlossen hat, benötigt für die Titelerteilung lediglich eine Teilnahmebestätigung über die absolvierte Facharztprüfung. Achtung: Sämtliche Voraussetzungen müssen innert zweier Jahre nach dem Inkraftsetzungstermin erfüllt sein (inkl. komplette Facharztprüfung, ausser wenn die Fachgesellschaft die Zulassung zum 2 . Teil wegen ungenügendem 1 . Teil verweigert)!

In folgenden Fachgebieten ist weiterhin nur die Teilnahme an der Facharztprüfung gefordert, mit anderen Worten: Das Bestehen der Prüfung ist nicht Voraussetzung für die Erteilung des Facharzttitels.

- Herz- und thorakale Gefässchirurgie;

- Kiefer- und Gesichtschirurgie;

- Klinische Pharmakologie und Toxikologie;

- Medizinische Genetik;

- Nephrologie. 


\begin{tabular}{|c|c|c|c|c|c|}
\hline \multicolumn{2}{|c|}{$\begin{array}{l}\text { Tabelle } \\
\text { Muss ich die Facharztprüfung bestehen? } \\
\text { (Fortsetzung von der vorhergehenden Seite) } \\
\text { Facharzttitel } \\
\text { Inkraftsetzungstermin }\end{array}$} & \multirow[t]{2}{*}{ 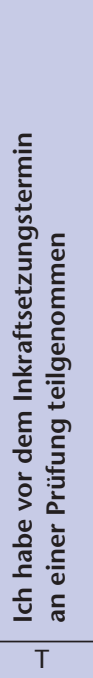 } & \multirow[t]{2}{*}{ 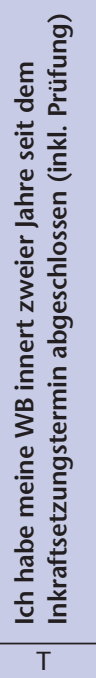 } & \multirow[t]{2}{*}{ 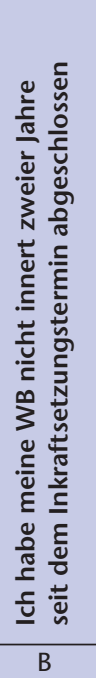 } & \multirow[t]{2}{*}{ 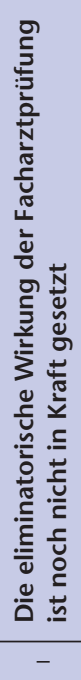 } \\
\hline ORL (ohne Hals- und Gesichtschirurgie) & 1.1 .2001 & & & & \\
\hline Orthopädische Chirurgie & 1.1 .2003 & $T$ & $T$ & B & - \\
\hline Pathologie & 1.1 .1999 & $\mathrm{~T}$ & $\mathrm{~T}$ & B & - \\
\hline Pharmazeutische Medizin & 1.1 .2003 & $T$ & $T$ & B & - \\
\hline Physikalische Medizin und Rehabilitation & 1.1 .2003 & $\mathrm{~T}$ & $\mathrm{~T}$ & B & - \\
\hline $\begin{array}{l}\text { Plastische, rekonstruktive und ästhetische } \\
\text { Chirurgie }\end{array}$ & 1.1 .2004 & $T$ & $\mathrm{~T}$ & B & - \\
\hline Pneumologie & 1.1 .2003 & $T$ & $T$ & B & - \\
\hline Prävention und Gesundheitswesen & 1.1 .2004 & $T$ & $T$ & B & - \\
\hline Psychiatrie und Psychotherapie & 1.1 .2001 & $T$ & $T$ & $B$ & - \\
\hline Radiologie & 2.4 .1986 & - & - & B & - \\
\hline Radio-Onkologie / Strahlentherapie & 2.4 .1986 & - & - & $B$ & - \\
\hline Rechtsmedizin & 1.1 .2001 & $T$ & $T$ & $B$ & - \\
\hline Rheumatologie & 1.1 .2001 & $\mathrm{~T}$ & $T$ & B & - \\
\hline Tropen- und Reisemedizin & 1.1 .2001 & $T$ & $T$ & B & - \\
\hline Urologie & 1.1 .2002 & $T$ & $T$ & B & - \\
\hline \multicolumn{6}{|l|}{ Schwerpunkte } \\
\hline Neonatologie & 1.1 .2003 & $\mathrm{~T}$ & $\mathrm{~T}$ & B & - \\
\hline Neuropädiatrie & 1.1 .2003 & $\mathrm{~T}$ & $\mathrm{~T}$ & B & - \\
\hline Pädiatrische Kardiologie & 1.1 .2001 & $\mathrm{~T}$ & $\mathrm{~T}$ & B & - \\
\hline Pädiatrische Endokrinologie-Diabetologie & 1.1 .2005 & $\mathrm{~T}$ & $\bar{T}$ & B & - \\
\hline alle anderen Schwerpunkte & & - & - & - & $\mathrm{T}$ \\
\hline \multicolumn{6}{|c|}{$\begin{array}{l}\text { B = Bestehen; } T=\text { Teilnehmen. } \\
\text { Die gesamte Weiterbildungsdokumentation (mit den aktuellen Prüfungsterminen) ist auf dem In- } \\
\text { ternet unter www.fmh.ch/awf abrufbar. Die Prüfungstermine werden auch in der Schweizeri- } \\
\text { schen Ärztezeitung publiziert. Bei allfälligen Rückfragen steht Ihnen das Sekretariat Aus-, Weiter- } \\
\text { und Fortbildung (AWF) der FMH gerne zur Verfügung. Adresse: Postfach 170, Elfenstrasse 18, } \\
3000 \text { Bern 15, Tel. } 03135911 \text { 11, Fax } 03135911 \text { 12, E-Mail: diplome@hin.ch. }\end{array}$} \\
\hline
\end{tabular}

